\title{
The r-Process in Metal Poor Stars and Black Hole Formation
}

\author{
R.N. Boyd \\ Physics and Life Sciences, L-050, Lawrence Livermore National Laboratory, Livermore, \\ CA 94550, USA \\ boyd11@llnl.gov \\ M.A. Famiano \\ Dept. of Physics and Joint Institute for Nuclear Astrophysics, Western Michigan \\ University, 1903 W. Michigan Avenue, Kalamazoo, MI 49008-5252, USA \\ B.S. Meyer \\ Dept. of Physics and Astronomy, Clemson University, 118 Kinard Laboratory, Clemson, \\ SC 29634-0978 USA \\ Y. Motizuki \\ RIKEN Nishina Center, Hirosawa 2-1, Wako, 351-0198 Japan \\ T. Kajino ${ }^{1}$ \\ National Astronomical Observatory of Japan, 2-21-1 Mitaka, Tokyo, 181-8588, Japan \\ and \\ I.U. Roederer \\ Carnegie Observatories, 813 Santa Barbara Street, Pasadena, CA 91101, USA
}

\begin{abstract}
Nucleosynthesis of heavy nuclei in metal-poor stars is generally thought to occur via the r-process because the r-process is a primary process that would have operated early in the Galaxy's history. This idea is strongly supported by the fact that the abundance pattern in many metal-poor stars matches well the inferred
\end{abstract}

\footnotetext{
${ }^{1}$ Dept. of Astronomy, Graduate School of Science; Univ. of Tokyo, 7-3-1, Hongo, Bunkyo-ku, Tokyo 113-0033, Japan
} 
Solar r-process abundance pattern in the mass range between the second and third r-process abundance peaks. Nevertheless, a significant number of metalpoor stars do not share this standard r-process template. In this Letter we suggest that the nuclides observed in many of these stars are produced by the r-process, but that it is prevented from running to completion in more massive stars by collapse to black holes before the r-process is completed, creating a "truncated r-process," or "tr-process." We find that the observed fraction of tr-process stars is qualitatively what one would expect from the initial mass function, and that an apparent sharp truncation observed at around mass 160 could result from a combination of collapses to black holes and the difficulty of observing the higher mass rare earths. We test the tr-process hypothesis with r-process calculations that are terminated before all r-process trajectories have been ejected. We find qualitative agreement between observation and theory when black hole collapse and observational realities are taken into account.

Subject headings: stars: Population II — nucleosynthesis — black hole physics

\section{Introduction}

The r-process has been understood for many years to (1) synthesize half the nuclides heavier than iron, (2) synthesize all of the nuclides heavier than ${ }^{209} \mathrm{Bi}$, and (3) be primary in the sense that its nucleosynthesis does not appear to depend on preexisting nuclides (Burbidge et al. 1957; Woosley et al. 1994; Wallerstein et al. 1997; Farouqi et al. 2009). In this latter context, its production of the heavier r-process nuclides in many metal poor stars appears to be very uniform (Sneden \& Cowan 2003), and it also produces relative rprocess abundances that are essentially the same as those found in more modern stars. A standard interpretation of the r-process suggests that it occurs in the neutrino wind that emanates from core-collapse supernovae (Woosley et al. 1994; Takahashi. Witti. \& Janka 1994; Farougi et al. 2009), although that interpretation is not without its issues (Qian \& Wooslev 1996; Mever, McLaughlin, \& Fuller 1998; Hudepohl, L. et al. 2010a,b; Fischer et al. 2010; Roberts, Woosley, \& Hoffman 2010). Even with these difficulties, though, there are a sufficient number of uncertainties, e.g., the possibility of sterile neutrinos (McLaughlin et al. 1999; McLaughlin et al. 2003), that this r-process site might turn out to be viable.

At the same time, other metal poor stars exhibit some of the features of r-process nucleosynthesis, but their abundances represent a surprisingly poor match with the "standard" abundance template (Aoki et al. 2000; Honda et al. 2007; Roederer et al. 2010a), perhaps most frequently identified as that observed in CS-22892-052 (Sneden \& Cowan 2003). A re- 
cent paper (Roederer et al. 2010a) has summarized the situation that exists for metal-poor stars. The data in that paper seem to suggest that a distribution of r-process abundances exists in metal poor stars, with some resembling the standard abundance set, but with a significant fraction of stars having abundances that do not match the standard template. These latter stars appear to favor the lighter r-process nuclides at varying levels, and many seem to have abundance patterns that terminate around Dy, that is, around mass 160.

In this Letter we point out that the abundance patterns observed for the stars that do not fit the standard r-process template could be produced by stars that are sufficiently massive that their core collapse first produce neutron stars but that the infall that occurs following the formation of the neutron star subsequently results in collapse to a black hole so called "fallback supernovae." This class of stars spans a mass range from roughly 25 to 40 solar masses (Heger et al. 1992) for low-metallicity stars. When the neutron star collapses to a black hole the r-process enrichment of the interstellar medium would cease, terminating either when the r-processed regions were swallowed by the black hole, or when the electron antineutrinos fell below the event horizon (Sasaqui, Kajino, \& Balantekin 2005). Thus, this truncated r-process, or tr-process, nucleosynthesis would terminate at different stages of that process, depending on the precise time at which the black hole prevented further r-process production or emission of nuclides into the interstellar medium. We suggest that the delayed collapse to the black hole, combined with another effect, namely, the difficulties in observing the higher mass rare earths, could produce the cutoff in the r-process distributions observed around $160 \mathrm{u}$.

The general scenario we envision merely assumes that mass elements that predominantly produce the lighter r-process nuclei are ejected before the mass elements that produce the heavier r-process nuclides. Any setting within a core-collapse supernova that satisfies this condition would allow for a truncated r-process. To evaluate this idea quantitatively, however, we apply the neutrino-driven wind model for the r-process. Although, as noted above, this model is not without issues; it is plausible and well discussed in the literature and makes a good setting for discussing the truncated r-process.

\section{Neutrino Wind Model of the r-Process}

In the neutrino wind scenario of the r-process, neutrinos from the nascent neutron star heat material at the neutron star surface and drive it away in a wind. As a wind element leaves the neutron star surface, it expands and cools. Nucleons in the wind element assemble into heavier nuclei, which serve as the seeds for the subsequent r-process (Woosley et al. 1994). Importantly, the abundance pattern for the seed nuclei is well-described by a quasi- 
equilibrium, which, for the entropy and neutron richness characteristic of neutrino-driven wind envirnoments, has a relatively sharp abundance peak near $A \sim 100$. As the temperature in the wind element falls further, charged-particle reactions freeze out. If a high neutron density accompanies the seed nuclei, neutron captures and beta decays successively promote the seed nuclei to higher mass. This upwards flow in mass slows at the neutron closed shells at 82 and then 126 neutrons, and thereby produces the r-process abundance peaks at A $\sim 130$ and $195 \mathrm{u}$ (Woosley et al. 1994; Wanajo et al. 2002).

For what follows, it is essential to note that the neutrino-driven wind leaves the star over a period of several seconds. In the standard scenario (Mever et al. 1992; Woosley et al. 1994; Takahashi. Witti, \& Janka 1994), the wind elements that leave the star early have lower entropy and a lower degree of neutron richness. As the neutron star evolves, the wind elements that leave later have higher entropy and a greater degree of neutron richness. It is in these latter wind elements that the heaviest nuclei are made. The lower-entropy, less neutron rich wind elements that leave the neutron star earlier produce ligher nuclei. If the neutron star collapses to a black hole after the lower-entropy elements leave the star but before the higher-entropy do, then the r-process has been truncated, and the abundance pattern will be dominated by lighter r-process nuclei.

Current supernova models do not naturally produce the parameters required to produce a successful r-process, most notably the entropy, but also the requisite neutron richness. While it may in fact be the case that core-collapse supernovae are not the site of the rprocess, it may also be that current supernova models are not sufficiently detailed to provide an adequate description of the r-process conditions in core-collapse events. For example, results from multidimensional hydrodynamics calculations suggest that the instabilities resulting from those calculations may ultimately be shown to produce the entropy required for making the heaviest r-process nuclei (Burrows et al. 2006). Interestingly, these instabilities may generate the neutron star kicks (Burrows et al. 2006; Guilet. Sato, \& Foglizzo 2010) that give rise to high space velocities of some pulsars (Arzoumanian et al. 2002). It will also be necessary to include all of the detailed neutrino physics (Burrows \& Thomson 2002; Roberts, Woosley, \& Hoffman 2010; Liebendoerfer et al. 2005) to adequately characterize the neutron richness in matter expelled from the supernova.

Future work will determine whether more advanced core-collapse supernova models will provide the conditions needed for the r-process. For the purposes of the present paper, we will simply assume that neutrino-driven winds in core-collapse supernovae are at least one of the sites of r-process nucleosynthesis. 


\section{Model Calculations}

To study the truncated r-process in the neutrino-driven wind, we applied the basic idea behind the study of Woosley et al. (1994), which assumed that the r-process occurred in the neutrino wind from a core collapse supernova. In that study, a succession of 40 "trajectories" (that is, thin shell wind elements), all assumed to have originated deep within the (assumed spherically symmetric) star, but having initially different conditions of density, temperature, entropy, and electron fraction, were emitted into the interstellar medium, thus contributing to the total r-process nucleosynthesis. The bubble was evolving in time, so that the conditions under which the individual trajectories were processed changed with the identity of the trajectory. We assumed that the different trajectories were emitted from the star successively, but ceased to be emitted when the collapse to the black hole occurred. This would be consistent with Woosley et al. (1994), who assumed successive emissions of the trajectories to generate what turned out to be a good representation of the Solar r-process abundances.

For our r-process calculations, we used a network code based on libnucnet, a library of $\mathrm{C}$ codes for storing and managing nuclear reaction networks (Mever \& Adams 2007). The nuclear and reaction data for the calculations were taken from the JINA reaclib database (Cyburt et al. 2010). We performed calculations for trajectories 24 - 40 in the Woosley et al. (1994) hydrodynamics model. For each trajectory, reaction network calculations were performed for $\mathrm{T}_{9}<2.5$ using initial abundances derived from the Woosley et al. (1994) results. Our calculations were simplified by assuming an initial abundance of massive nuclei from a single nucleus heavy seed with a mass $\mathrm{A}$ equal to the average mass at $\mathrm{T}_{9}=2.5$ and an atomic number derived from the average mass number, the $\mathrm{Y}_{e}$, and the neutron and alpha mass fractions at $\mathrm{T}_{9}=2.5$ in Woosley et al. (1994). We justify this by noting that the heavynuclide distribution is typically sharply peaked near $\mathrm{T}_{9}=2.5$. An adiabatic expansion was assumed for each trajectory in the nucleosynthesis, with entropy constant within a trajectory but varying between trajectories, again consistent with the approach of Woosley et al. (1994) for times at which the temperature $\mathrm{T}_{9}<2.5$. The material was taken to expand at constant velocity on a timescale consistent with that derived from Woosley et al. (1994). For each trajectory the calculation was continued until neutron capture reactions had ceased and the abundance distribution versus mass number had frozen out. Our representation of the full r-process abundances, shown as the dots in Figure 1, is not as good as that achieved by Woosley et al. (1994), but our calculations do produce the basic features of the full r-process, namely the mass 130 and $195 \mathrm{u}$ peaks.

The simulation of Woosley et al. (1994) that produced a good r-process representation began with trajectory number 24 and summed the nucleosynthesis yields from the remain- 
ing 16 elements since all of these trajectories would have been ejected into the interstellar medium. We also began with trajectory 24, and performed a mass weighted sum of the nucleosynthesis from the trajectories up to some higher number trajectory to observe the resulting nucleosynthesis when the trajectories beyond our maximum trajectory were assumed to be consumed by the collapse to a black hole. This produced a result similar to that of Woosley et al. (1994) when all trajectories from 24 to 40 were included. The results are shown in Figure 1. There it can be seen that truncating the r-process at increasing trajectory number does terminate the r-process nucleosynthesis at increasingly higher nuclear mass. Note that although the curve representing trajectories 24 through 31 does reach the mass 195 u peak, the abundance produced in that calculation is nearly two orders of magnitude below that of the full r-process, which would render the higher mass nuclides difficult to observe. The abundances for that calculation would therefore appear observationally to terminate at a mass of about $140 \mathrm{u}$.

In Figure 2 we compare several tr-process calculations with the derived elemental abundance pattern in the metal poor halo star HD 122563. This low-metallicity star $([\mathrm{Fe} / \mathrm{H}]=$ -2.7) is deficient in the heavy neutron-capture elements ( $\mathrm{Ba}$ and heavier) relative to the light neutron-capture elements (Sr through $\mathrm{Cd}$ ) when compared with the scaled Solar r-process pattern. The HD 122563 abundances are a very poor match to the scaled Solar s-process pattern. Its abundance pattern matches the scaled Solar r-process pattern better up to an atomic mass of about 70 (mass of $\sim 174 \mathrm{u}$ ), but even this fit is unsatisfying (Sneden \& Parthasarathy 1983; Honda et al. 2006). Stars like HD 122563 may be candidates for enrichment by the tr-process. Figure 2 demonstrates that the tr-process predictions, while far from a perfect match to the individual abundances, can reproduce the relative downward trend in abundance with increasing atomic number seen in some metal poor stars. More exploration of tr-process calculations is obviously needed, but the general trend is encouraging.

\section{Probability of Occurence of tr-Process Stars}

In principle, a test of the tr-process model would be provided by a large set of data for metal poor stars that spans the masses of the nuclides produced in the r-process from mass $\sim 80$ to the heaviest observable nuclides that the r-process synthesizes, usually thorium. Unfortunately this is challenging due to the difficulty of observing the higher mass rare-earth nuclides, starting at mass $\sim 160$. Thus all $\mathrm{r}$-process events that terminate at masses between 160 and lead would appear to terminate at mass 160, producing the effect observed by Roederer et al. (2010a).

Any standard initial mass function (IMF) reflects the fact that less-massive stars are 
more numerous than more-massive stars. Assuming that stars from 8 to 40 solar masses may produce comparable amounts of r-process material, we can use the IMF to estimate the fraction of stars whose r-process may be truncated by collapse to a black hole. We adopt the Salpeter (1954) IMF, for which $\mathrm{dN} / \mathrm{dm} \propto \mathrm{m}^{-2.35}$ for massive stars, where $\mathrm{m}$ is the stellar mass in units of the solar mass, and $\mathrm{N}$ is the number of stars of a given mass per unit volume. The ratio of the number of stars that would be expected to collapse to black holes (25-40 solar masses) to those expected to collapse to neutron stars (8-25 solar masses) is 0.13 in a well-sampled IMF.

While a number of r-process rich stars (here taken to mean stars with $[\mathrm{Eu} / \mathrm{Fe}]>+1.0$ ) have been discovered and studied in detail in the last two decades, these stars constitute a relatively minor fraction of all metal-poor stars. More unbiased samples (McWilliam et al. 1995; Barklem et al. 2005) find that stars with $[\mathrm{Eu} / \mathrm{Fe}]>+1.0$ comprise $<10 \%$ of all stars with $[\mathrm{Fe} / \mathrm{H}]<-2.0$. Figure 11 of Roederer et al. (2010a) suggests that stars with $[\mathrm{Eu} / \mathrm{Fe}]$ $<0$ are candidates for enrichment by a truncated r-process. Difficulties in detecting Eu in metal-poor stars with $[\mathrm{Eu} / \mathrm{Fe}]<0$ make it even more challenging to estimate how numerous these stars are. $\mathrm{Ba}$ is more easily detected and may be used to represent Eu and other heavy elements in stars lacking s-process enrichment. From the Ba abundances in the large survey of Barklem et al. (2005) we estimate a lower limit of $\sim 55 \%$ of metal-poor stars as candidates for enrichment by a truncated r-process. This is significantly higher than the $13 \%$ derived above assuming a Salpeter IMF, yet Figure 2 demonstrates that a tr-process may be one way to produce the heavy nuclides observed in some metal-poor stars. The discrepancy in these fractions could indicate that additional nucleosynthesis channels, such as those proposed by, e.g., Qian \& Wasserburg (2008), Farouqi et al. (2009), Wanajo et al (2011), or Nakamura et al. (2011), together with the tr-process may contribute to the chemical enrichment of the early Galaxy. Given that massive stars with short lifetimes will dominate the chemical enrichment at early times, though, the tr-process may very well have been a major source of heavy nuclei at these epochs.

\section{Other r-Process Issues}

As a possible additional benefit of the tr-process, we note that the stars that were truncated at mass points 28 or less produced no nuclides in the mass 130 peak or beyond. This would have the effect of boosting the yields of the lighter r-process nuclides relative to the heavier ones, filling in the mass 110 to $120 \mathrm{u}$ region. It has been known for some time that r-process calculations that produce a mass $195 \mathrm{u}$ peak underproduce the nuclides in the 110-120 mass region. The r-process nuclides that were even lighter than those were 
underproduced by an even larger factor, but this would also be consistent with distributed cutoffs of the r-process nucleosynthesis. Thus, again guided by the IMF, but along with the mass dependent cutoff times, the tr-process might potentially solve this troublesome aspect of current r-process analyses (Kratz et al. 2007; Farouqi et al. 2009). Conclusions in this regard, however, must await more detailed analyses.

An obvious test of the tr-process model could occur from renewed effort to observe the higher mass rare earth elements in very metal poor stars with low [Eu/Fe] ratios. If elements in that mass region can be observed, and the tr-process is correct, additional observations would be expected to map out the black hole collapse times over that region. While no doubt challenging, a relationship between observations and black hole collapse times would be of great interest.

RNB's work has been supported under the auspices of the Lawrence Livermore National Security, LLC (LLNL) under Contract No. DE-AC52-07NA27344; MF's by National Science Foundation grant PHY-0855013; BSM's by NASA grant NNX10AH78G; YM's by the NEXT Program of JSPS and CSTP (GR098); TK's by Grants-in-Aid for Scientific Research of JSPS (20244035), Scientific Research on Innovative Area of MEXT (20105004), and Heiwa Nakajima Foundation; and IUR's by the Carnegie Institution of Washington through the Carnegie Observatories Fellowship. This is document LLNL-JRNL-491647.

\section{REFERENCES}

Aoki, W., Norris, J.E., Ryan, S.G., Beers, T.C., \& Ando, H. 2000, ApJ, 536, L97

Arzoumanian, Z., Chernoff, D.F., \& Cordes, J.M. 2002, ApJ, 568, 289

Barklem, P.S., Christlieb, N., Beers, T.C., Hill, V., Bessell, M.S., Holmberg, J., Marsteller, B., Rossi, S., Zickgraf, F.-J., \& Reimers, D. 2005 A\&A, 439, 129

Burbidge, M., Burbidge, G., Fowler, W.A., \& Hoyle, F. 1957, Rev. Mod. Phys., 29, 547

Burrows, A. \& Thomson, T.A. 2002, Core Collapse of Massive Stars, ed. by C.L. Fryer, Kluwer Academic Publ.

Burrows, A., Livne, E., Dessart, L., Ott, C.D., \& Murphy, J. 2006, ApJ, 640, 878

Cyburt, R. H., Amthor, A. M., Ferguson, R., Meisel, Z., Smith, K., Warren, S., Heger, A., Hoffman, R. D., Rauscher, T., Sakharuk, A., Schatz, H., Thielemann, F. K., \& Wiescher, M., ApJS, 189, 240 
Farouqi, K., Kratz, K.-L., Pfeiffer, B., Rauscher, T., Thielemann, F.-K., \& Truran, J.W. 2009, ApJ, 694, L49

Fischer, T., Whitehouse, S.C., Mezzacappa, A., Thielemann, T.-K., \& Liebendorfer, M., 2010, A\&A, 517, A80

Guilet, J., Sato, J., \& Foglizzo, T. 2010, ApJ, 713, 1350

Heger, A., Fryer, C.L., Woosley, S.E., Langer, N., \& Hartmann, D.H. 1992, ApJ, 591, 288

Hoffman, R.D., Woosley, S.E., \& Qian, Y.-Z. 1997, ApJ, 482, 951

Honda, S., Aoki, W., Ishimaru, Y., Wanajo, S., \& Ryan, S.G. 2006, ApJ, 643, 1180

Honda, S., Aoki, W., Ishimaru, Y., \& Wanajo, S. 2007, ApJ, 666, 1189

Hudepohl, L., Muller, B., Janka, H.-T., Marek, A., \& Raffelt G.G. 2010, Phys. Rev. Lett., 104,251101

Hudepohl, L., Muller, B., Janka, H.-T., Marek, A., \& Raffelt G.G. 2010, Phys. Rev. Lett., 105, 249901

Käppeler, F., Beer, H., \& Wisshak, K. 1989, Rep. Prog. Phys., 52, 945

Kratz, K.-L., Farouqi, K., Pfeiffer, B., Truran, J.W., Sneden, C., \& Cowan, J.J. 2007, ApJ, 662,39

Liebendoerfer, M., Rampp, M., Janka, H.-Th. \& Mezzacappa, A. 2005, ApJ, 620, 840

McLaughlin, G.C., Fetter, J.M., Balantekin, A.B., \& Fuller, G.M. 1999, Phys. Rev. C, 59, 2873

McLaughlin, G.C., Fetter, J.M., Balantekin, A.B., \& Fuller, G.M. 2003, Astropart. Phys., 18,433

McWilliam, A., Preston, G.W., Sneden, C., \& Searle, L. 1995, Astron. J., 109, 2757

Meyer, B.S. \& Adams, D. C. 2007, Met. Planet. Sci., 42, 5125; see also http://sourceforge.net/projects/libnucnet/

Meyer, B.S. \& Brown, J. S. 1997, ApJS, 112, 199

Meyer, B.S., Mathews, G.J., Howard, W.M., Woosley, S.E., \& Hoffman, R.D. 1992, ApJ, 399, 656 
Meyer, B.S., McLaughlin, G.C., \& Fuller, G.M. 1998, Phys. Rev. C, 58, 3696

Nakamura, K., Sato, S. Harikae, S., Kajino, T., \& Mathews, G.J. 2011, ApJ (submitted)

Otsuki, K., Tagoshi, H., Kajino, T., \& Wanajo, S. 2000, ApJ, 533, 424

Qian, Y.-Z. \&Wasserburg, G.J. 2008, ApJ, 687, 272

Qian, Y.-Z. \&Woosley, S. E., ApJ, 471, 331

Roberts, L.F., Woosley, S.E., \& Hoffman, R.D. 2010, ApJ (submitted)

Roberts, L., \& Woosley, S.E., private communication

Roederer, I.U., Kratz, K.-L., Frebel, A., Christlieb, N., Pfeiffer, B., Cowan, J.J., \& Sneden, C. 2009, ApJ, 698, 1963

Roederer, I.U., Cowan, J.J., Karakas, A.I., Kratz, K.-L., Lugaro, M., Simmerer, J., Farouqi, K., \& Sneden, C. 2010a, ApJ, 724, 975

Roederer, I.U., Sneden, C., Lawler, J.E., \& Cowan, J.J. 2010b, ApJ, 714, L123

Salpeter, E.E. 1954, Australian J. Phys., 7, 373

Sasaqui, T., Kajino, T., \& Balantekin, A.B. 2005, ApJ, 634, 534

Schorck, T., et al. 2009, A\&A, 507, 817

Sneden, C. \& Parthasarathy, M. 1983, ApJ, 267, 757

Sneden, C., Cowan, J.J., Lawler, J.E. Ivans, I.I. Burles, S., Beers, T.C., Primas, F., Hill, V., Truran, J.W., Fuller, G.M., Pfeiffer, B., \& Kratz, K.-L. 2003, ApJ, 591, 936

Sneden C. \& Cowan, J.J. 2003, Science, 299, 70

Takahashi, K., Witti, J., \& Janka, H.-Th. 1994, A\&A, 286, 857

Wallerstein, G. et al. 1997, Rev. Mod. Phys., 69, 995

Wanajo, S., Itoh, N., Ishimaru, Y., Nozawa, S., \& Beers, T. 2002, ApJ, 577, 853

Wanajo, S., Janka, H.-J., \& Kubono, S. 2011, ApJ, 729, 46

Woosley, S.E., Wilson, J.R., Mathews, G.J., Hoffman, R.D., \& Meyer, B.S. 1994, ApJ, 433, 229 


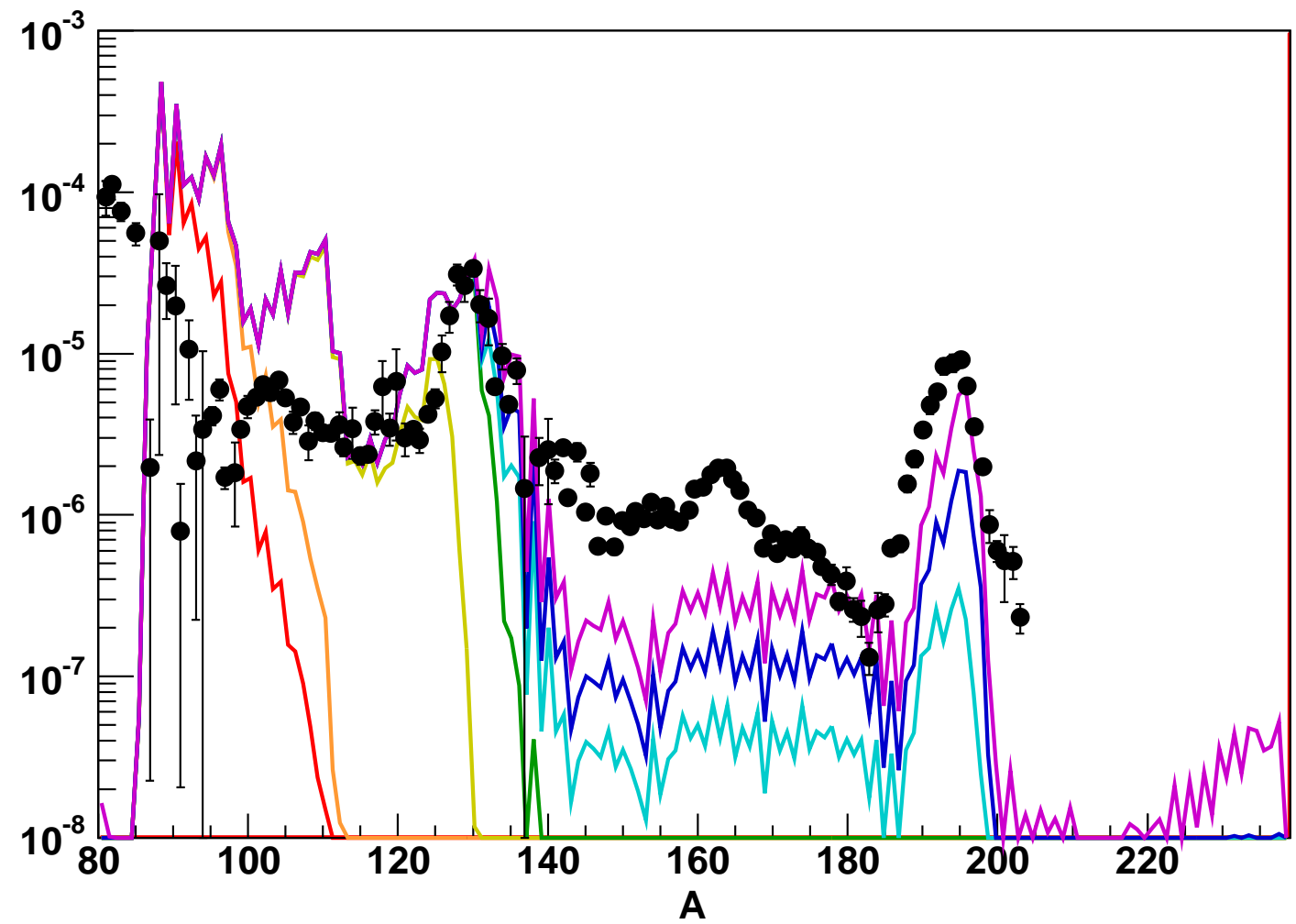

Fig. 1.- r-Process calculations using the trajectories from Woosley et al. (1994), but summing the results from trajectory number 24 to some later trajectory, as was done in Woosley et al. (1994). The successive curves include trajectory 24, 24 through 26, 24 through 28, 24 through 30, 24 through 31, 24 through 32, and 24 through 40, where the emission times of the latest trajectory in each sum are as indicated. The sums to higher number trajectories make both the mass 130 and 195 r-process peaks, and the sum through trajectory 40 comes closest to representing the Solar r-process abundances (Käppeler. Beer. \& Wisshak 1989), shown as the dots. 


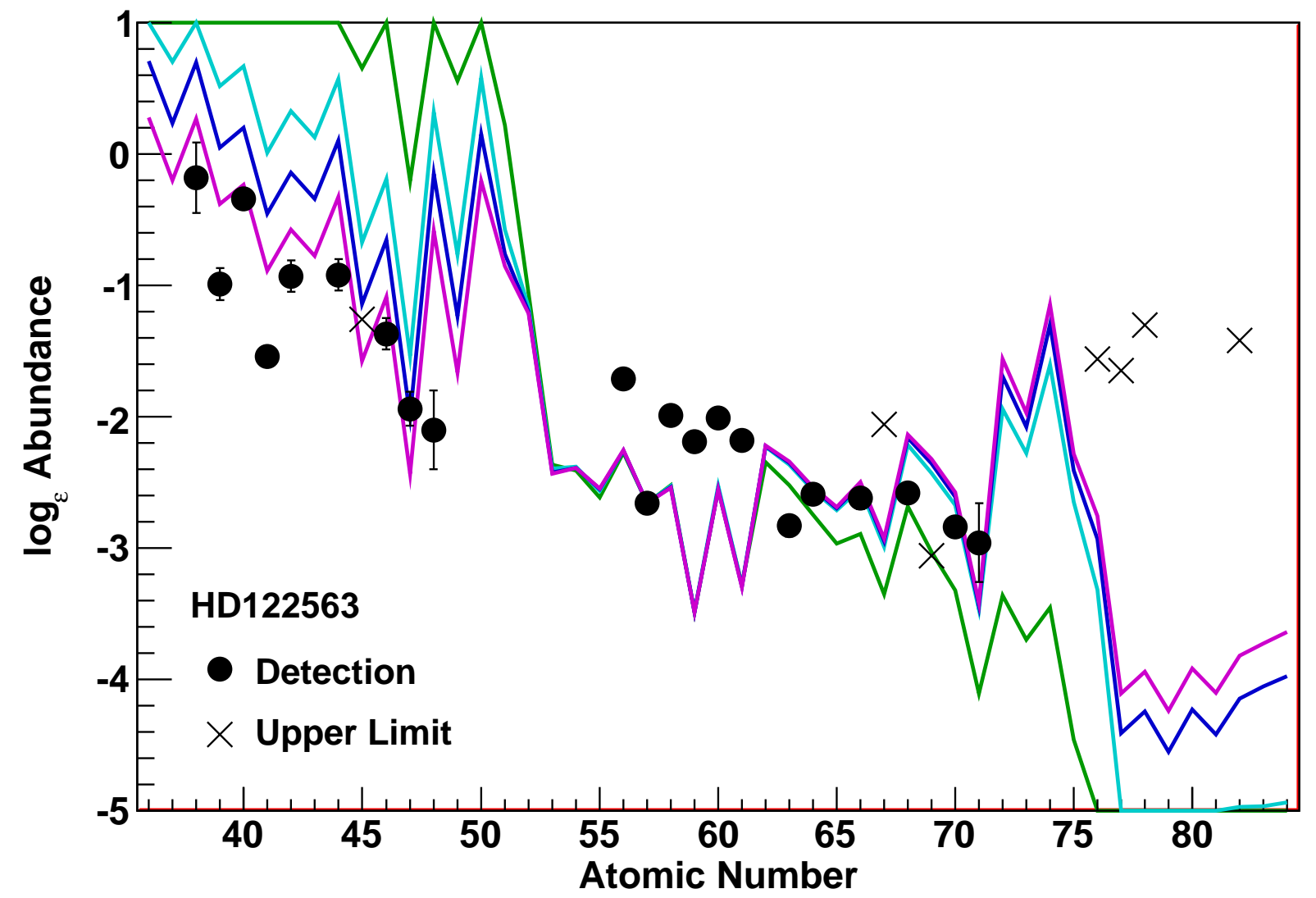

Fig. 2.- Comparison of tr-process predictions, using the formalism adapted from Woosley et al. (1994), for four mass cuts. These are cuts below shell 30 (green), 31 (light blue), 32 (dark blue), and 40 (purple) with abundances in the metal-poor star HD 122563. Observational data are from Honda et al. (2006) and Roederer et al. (2009, 2010b). The predictions are scaled to the La abundance $(\mathrm{Z}=57)$. 